\title{
Multimodal biometrics of fingerprint and signature recognition using multi-level feature fusion and deep learning techniques
}

\author{
Arjun Benagatte Channegowda ${ }^{1}$, H N Prakash ${ }^{2}$ \\ ${ }^{1}$ Department of Information Science and Engineering, Rajeev Institute of Technology (RIT), Affiliated to Visvesvaraya \\ Technological University (VTU), Karnataka, India \\ ${ }^{2}$ Department of Computer Science and Engineering, Rajeev Institute of Technology (RIT), Vis vesvaraya Technological \\ University (VTU), Karnataka, India
}

\begin{tabular}{l}
\hline \hline Article Info \\
\hline Article history: \\
Received Dec 10, 2020 \\
Revised Mar 1, 2021 \\
Accepted Mar 12, 2021 \\
\hline
\end{tabular}

Keywords:

Deep Neural Network

Feature Fusion

Fingerprint

Multimodal Biometrics

Signature

\begin{abstract}
Providing security in biometrics is the major challenging task in the current situation. A lot of research work is going on in this area. Security can be more tightened by using complex security systems, like by using more than one biometric trait for recognition. In this paper multimodal biometric models are developed to improve the recognition rate of a person. The combination of physiological and behavioral biometrics characteristics is used in this work. Fingerprint and Signature biometrics characteristics are used to develop a multimodal recognition system. Histograms of oriented gradients (HOG) features are extracted from biometric traits and for these feature fusions are applied at two levels. Features of Fingerprint and Signatures are fused using concatenation, sum, max, min, and product rule at multilevel stages, these features are used to train deep learning neural network model. In the proposed work, Multi-level feature fusion for multimodal biometrics with a deep learning classifier is used and results are analyzed by a varying number of hidden neurons and hidden layers. Experiments are carried out on SDUMLA-HMT, machine learning and data mining lab, Shandong University fingerprint datasets, and MCYT signature biometric recognition group datasets, and encouraging results were obtained.
\end{abstract}

This is an open access article under the CC BY-SA license.

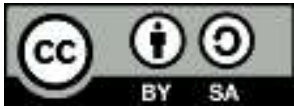

\section{Corresponding Author:}

Arjun B C

Department of Information Science and Engineering

Visvesvaraya Technological University (VTU)

Rajeev Institute of Technology, B M Bypass road, Industrial area, Hassan-573201, Karnataka, India

Email: bc.arjun@gmail.com

\section{INTRODUCTION}

In today's world, almost all security systems are using biometric recognition techniques. Biometric recognition has become the day-to-day activity for a bigger population. Daily usage like a mobile phone to bank locker all the systems are integrated with biometric security. In the coming days, these biometric recognition systems will be in almost all the areas of our day-to-day activity. By increasing usage of such a system with an increase in population, recognition will become more complex day by day. A Lot of research is going on multimodal biometric recognition. Since by using multimodal biometric traits more secured system can be developed over a unimodal biometric system [1]. When it comes to biometric traits two factors are considered, one is an extrinsic feature and another one intrinsic feature. The Extrinsic features can be visible from the outside and these features can be harmed by external factors [2]. Example fingerprint, face, etc. can be harmed in the accident. The Intrinsic features cannot be visible from the outside and these features cannot be harmed by external factors. Example finger vein, palm vein, etc. And biometric traits are broadly 
classified as physiological and behavioral biometrics characteristics, where the face, fingerprint, iris, etc. fall under physiological characteristics and signature, voice and gait. fall under behavioral biometrics characteristics. The Biometric recognition system is designed using four components they are, sensor module, feature extraction module, matching module, and decision-making module. Biometric recognition systems are categorized into two systems one is unimodal and another is a multimodal biometric recognition system. Unimodal uses single biometric traits where multimodal uses more than one biometric trait. Some of the unimodal biometric systems are explored in previous work on face and signatures biometric traits [3, 4]. In a multimodal system, a fusion of more than one biometric trait could be explored. Fusion could be explored at different levels. Fusion fall under four different levels they are sensor, feature, score, and decision. Recent research is more attractive towards the use of the deep learning method since it can handle limitations that are facing by machine learning techniques [5-7]. In the proposed work, a new multimodal biometric recognition modal is explored, which uses multi-level HOG feature fusion of signature and fingerprint with deep learning algorithm for recognition. Convolution neural networks (CNNs) [8] are designed and a softmax classifier is used for image classification. Adam optimization and categorical crossentropy were used as optimizer and loss function respectively.

\section{LITERATURE SURVEY}

A Lot of research is going on for decades on unimodal and multimodal biometrics recognition. In the past few years due to the advancement of technology in computer hardware, deep learning concepts are gaining more interest in research. Using the deep learning technique biometric recognition and verification showed better results [9] shown in Table 1. In this paper fingerprint and signature, biometric traits are considered, so previous works on fingerprint and signature biometric recognition and verification are compared in both unimodal and multimodal systems.

Table 1. Previous work on Multimodal Biometrics identification using deep learning

\begin{tabular}{|c|c|c|c|}
\hline Title & Deep Learning Architecture & Dataset & Results \\
\hline $\begin{array}{l}\text { Multimodal Face Biometrics by Using } \\
\text { Convolutional Neural Networks [10] }\end{array}$ & Self-designed CNN & Multi-PIE & $98.35 \%$ accuracy \\
\hline $\begin{array}{l}\text { Audio-VisualSpeaker Recognition via Multi- } \\
\text { modal Correlated Neural Networks [11] }\end{array}$ & Self-designed CNN & $\begin{array}{l}\text { Videos from Tv } \\
\text { series naming } \\
\text { "Friends." }\end{array}$ & $97.85 \%$ accuracy \\
\hline $\begin{array}{l}\text { A Novel Multi-Model BiometricFusion Approach } \\
\text { Using Palm-Print \& Face Biometric [12] }\end{array}$ & $\mathrm{NN}$ and SVM & Sample Images & $\begin{array}{l}\text { Cumulative match } \\
\text { score good results. }\end{array}$ \\
\hline $\begin{array}{l}\text { Multimodal BiometricAuthentication Using Back } \\
\text { Propagation Artificial Neural Network [13] }\end{array}$ & ANN classification & Sample Images & $98.34 \%$ accuracy \\
\hline $\begin{array}{l}\text { Multimodal Feature Level Fusion based on } \\
\text { Particle Swarm Optimization with Deep Transfer } \\
\text { Learning [14] }\end{array}$ & Modified VGG & $\begin{array}{l}\text { NICE.II database } \\
\text { Competition }\end{array}$ & $5.55 \% \mathrm{EER}$ \\
\hline $\begin{array}{l}\text { FKP and Iris BasedMultimodal Biometric System } \\
\text { Using PCA with NFNN [15] }\end{array}$ & $\begin{array}{l}\text { Neuro-Fuzzy Neural Networks } \\
\text { (NFNN) }\end{array}$ & $\begin{array}{l}\text { PolyU FKP and } \\
\text { CASIA } \\
\text { Iris database }\end{array}$ & EER of $0.23 \%$ \\
\hline $\begin{array}{l}\text { Score fusion offinger vein and face for human } \\
\text { recognition based onconvolutional neural network } \\
\text { model [16] }\end{array}$ & Traditional CNN feature extraction & $\begin{array}{l}\text { VERA } \\
\text { Fingervein, } \\
\text { Color Feret and } \\
\text { AR face database }\end{array}$ & $99.89 \%$ accuracy \\
\hline $\begin{array}{l}\text { Periocular biometrics for non-ideal images: with } \\
\text { off-the-shelf Deep CNN \& Transfer } \\
\text { Learningapproach [17] }\end{array}$ & $\begin{array}{l}\text { Pre-trained Alex net, Googlenet, } \\
\text { Resnet18, Resnet50, Resnet101, } \\
\text { VGG16 and VGG19 }\end{array}$ & UBIPr database & $\begin{array}{l}\text { Max. testing } \\
\text { accuracy of } 96 \% \\
\text { with VGG19 }\end{array}$ \\
\hline
\end{tabular}

From the above literature survey, it has been observed that deep learning techniques will improve multimodal biometric recognition. And not more work is done on fingerprint and signature biometric combinations for larger data sets. In this paper new modal is proposed where Multi-level feature fusion is done on multimodal biometrics [18] of fingerprint and signature traits using deep learning architecture.

\section{DATABASE USED FOR EXPERIMENTATION}

\subsection{Fingerprint database}

SDUMLA-HMT fingerprint database from the machine learning and data mining lab, Shandong University (SDUMLA) [19] is used for experimentation, 40 users with 6 fingerprint samples of the left thumb of each person are considered for experimentation. Fingerprint samples are shown in Figure 1. 


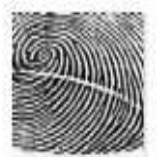

1.bmp

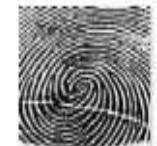

2.bmp

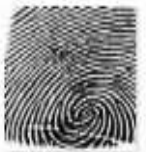

$3 . \mathrm{bmp}$

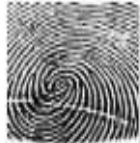

4.bmp

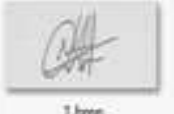

15me

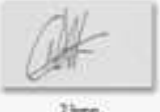

2 bis

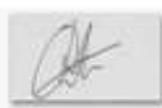

$36 \mathrm{me}$

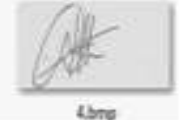

4

SDUMIA-HMT Person-1 left thumb samples.

Figure 1. SDUMLA Fingerprint Image Samples

Figure 2. MCYT data base signature samples

\subsection{Offline signature database}

MCYT Biometric Recognition Group-ATVS database [20] is used for experimentation, out of 75 users, 40 users with all genuine 6 signatures are considered for experimentation. Signature samples are shown in Figure 2.

\section{PROPOSED ARCHITECTURE}

In the proposed work a novel Multi-level feature fusion modal for multimodal biometrics fingerprint and signature traits developed as shown in Figure 3. From a standard database upsampled database is created using the bilinear interpolation technique [21]. After creating a new upsampled database, HOG features [22] are extracted from both standard and upsampled databases separately, these separate HOG features are fused together using SUM, MAX, MIN, PRODUCT, and concatenation method in feature fusion level 1. Fingerprint and Signature fused HOG feature vectors are stored separately shown in Figure 4. In level 2 feature fusion, HOG features of fingerprint and signatures are fused using the concatenation method respectively for each fused sample from level 1 [23]. The Final HOG feature vector obtained after level 1 and level 2 fusion is set as an input to neural network.

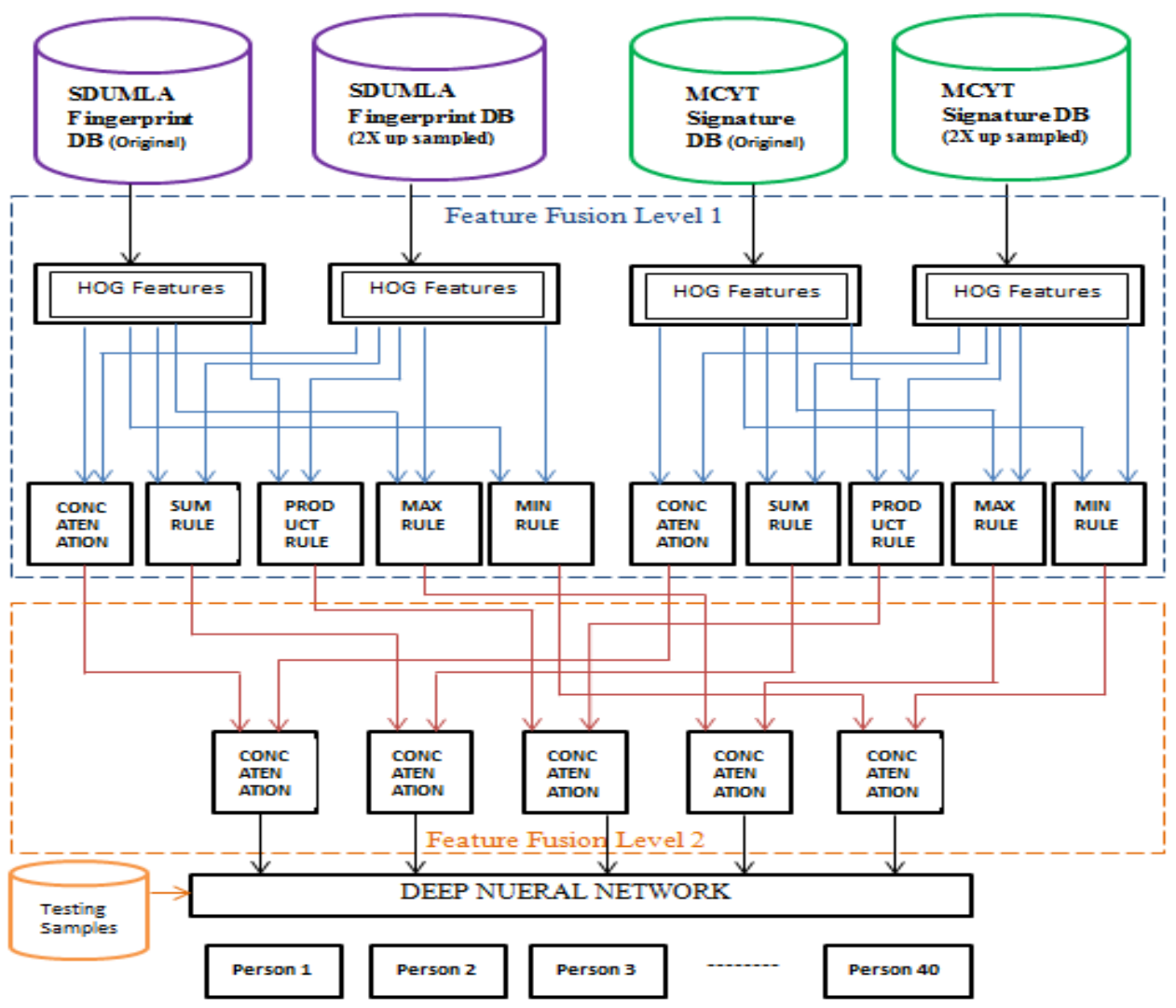

Figure 3. Multi-level multimodal biometric fusion of fingerprint and signature biometric traits 


\begin{tabular}{|c|c|c|c|c|c|c|c|}
\hline 0.06655 & 0.06226 & 0.06285 & 0.07780 & 0.11301 & 0.10340 & $\ldots$ & Up to 180 columns \\
\hline \multicolumn{8}{|c|}{ HOG features of Person 1 up sampled signature: } \\
\hline 0.07424 & 0.06960 & 0.07265 & 0.08779 & 0.13855 & 0.10069 & $\ldots+\ldots$ & Up to 180 columns \\
\hline \multicolumn{8}{|c|}{ Level 1 fusion using max rule for Person 1 signature } \\
\hline 0.07424 & 0.06960 & 0.07265 & 0.08779 & 0.13855 & 0.10340 & +क & Up to 180 columns \\
\hline
\end{tabular}

Figure 4. Signature feature samples of person 1

Level 1 fusion is carried out with SUM, MAX, MIN, PRODUCT and concatenation method and in level 2 concatenation method is applied.

$$
\begin{aligned}
& \text { SUM operation: } \mathrm{Cij}=\mathrm{Aij}+\mathrm{Bij} \\
& \text { MAX operation: } \mathrm{Cij}=\mathrm{MAX}(\mathrm{Aij}, \mathrm{Bij}) \\
& \text { MIN operation: } \mathrm{Cij}=\mathrm{MIN}(\mathrm{Aij}, \mathrm{Bij}) \\
& \text { Product operation: } \mathrm{Cij}=\mathrm{Aij} * * \mathrm{Bij} \\
& \text { Concatenation: } \mathrm{Cij}=[\mathrm{Aij} \mathrm{Bij}]
\end{aligned}
$$

Where Aij is HOG feature vector of standard signature and Bij is HOG feature vector of upsampled signature. Similar steps carried out for fingerprint data sets.

\section{THE NEURAL NETWORK MODEL}

In this paper using Python with Keras Library Deep learning neural networks are created. by following the model life-cycle steps deep learning neural networks are created [24]. Steps as follows define network, compile network, Fit network, evaluate network and make predictions.

Networks are defined in sequence order

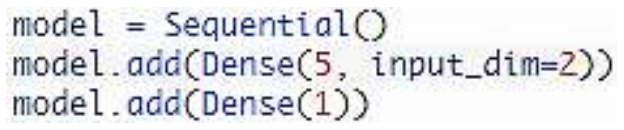

Consider the example Input dim are several input neurons, value 5 is a number of hidden neurons, and an output neuron is 1 . In this paper more than 100 input neurons, more than 500 hidden neurons, and 40 neurons as output neurons, since 40 different multiclass classifications are expected as output.

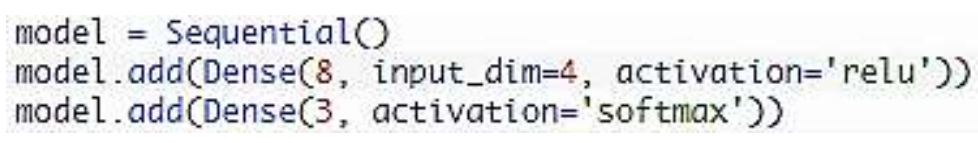

Since the proposed work is explored on multiclass classification softmax activation function is used at the output layer. Complex deep learning models are also defined and explored in this work using more than one hidden layer.

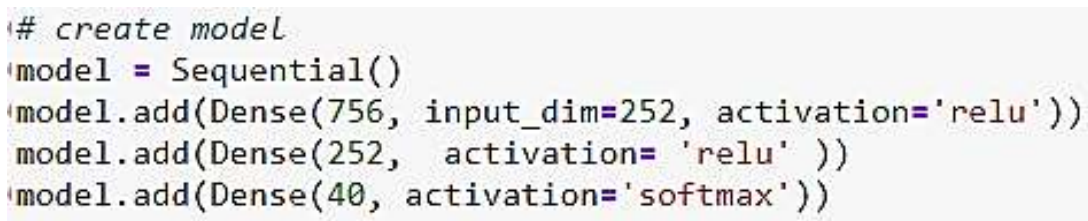

In this paper, 252 input neurons with 756 hidden neurons in the first hidden layer and 252 hidden neurons in the second hidden layer, and an output layer with 40 neurons are used in one of the case studies. Compile network: Multiclass logarithmic loss or categorical cross-entropy is used with the 'adam' optimization algorithm. Fit network: Model batch size and epochs are assigned to fit the model in this step. After this step evaluation of the model is done by checking classification accuracy and finally, predictions for new data sets are executed. 


\section{EXPERIMENTS}

In this paper, the main aim is to improve the recognition rate of fingerprint and signature biometric traits for larger data sets. SDUMLA-HMT fingerprint database and MCYT biometric recognition group signature database are used for experimentation. Fused features are used to train and test the deep learning neural network. An example of the fused feature $[25,26]$ using concatenation technique is:

Features fusion: Consider a example of fused feature, $\mathrm{X} 1$ is a HOG feature of fingerprint and Y1 is a HOG feature of signature, after concatenation output is $\mathrm{Z}$, therefore, first three samples of each person give Z1 to Z40, forty rows with column (number of columns depends on fusion technique used) and last column with the class label name data matrix are created, this data matrix is used to train the deep learning neural network. Similarly, for the testing phase, the same procedure for the remaining three data samples without a class label column is used for experimentation.

$$
\begin{array}{r}
\text { Concatenation Technique- } X_{1}=\left[X_{1}, X_{2} \ldots X_{36}\right] Y_{1}=\left[Y_{1}, Y_{2} \ldots Y_{36}\right] \\
Z=\left[X_{1}, X_{2} \ldots . X_{36}, Y_{1}, Y_{2} \ldots . Y_{36}\right] \\
Z_{1}=\left[X_{1}, X_{2} \ldots X_{36}, Y_{37}, Y_{38} \ldots . . Y_{72+1}\right]
\end{array}
$$

a. Unimodal biometrics: Initial experiments were explored on unimodal systems, where HOG features are extracted from the standard database and upsampled database of fingerprint and signature separately. Unimodal HOG features [27] are fused using SUM, MAX, MIN, PRODUCT, and concatenation method, and fused feature vector from (1) (2) of each method is set as input to the neural network.

b. Multimodal biometrics: Experiments are carried out on multimodal biometric recognition where features fused while executing unimodal experiments are considered from fingerprint and signature biometrics, these features are fused once again using the concatenation method, and results are plotted. These multilevel Fingerprint and Signatures are fused using concatenation, sum, max, min, and product rule at multilevel stages as shown in Figure 4, these features are used to train deep learning neural network model. Example, After fusion from (1) (2) of HOG fingerprint features of 3 training samples and HOG signature features of 3 training samples, data with class label name in the last column is used to train deep learning neural network, remaining 3 samples of fingerprint and signatures are fused and data without label name is used for testing the deep learning network gives the output class name and classification accuracy is analyzed.

c. Deep learning model: In the proposed work, by varying input neurons, hidden neurons, and hidden layers different deep learning models are designed and explored. Deep learning models were designed using Keras libraries using python.

d. Experiments of unimodal system: The MCYT signature data sets consist of 75 users with 15 genuine and 15 forgery signatures. For this experiment, 40 people with 6 data samples of each person are used. HOG features of the first 3 samples of each person's Standard signature data sets and 1:2 ratio upsampled signature data sets are fused using SUM, MAX, MIN, PRODUCT, and concatenation techniques. The neural network model is designed for the required input. The remaining 3 samples of each person are used for testing. The same procedure is executed for the fingerprint database, here 40 user's left thumb 6 data samples of each user are considered.

e. Experiments of multimodal system: The feature vector obtained in level 1 after SUM, MAX, MIN, PRODUCT, and concatenation technique of Unimodal system is used for the second level of fusion. Feature vector obtained from each technique in level 1 of both signature HOG features and Fingerprint HOG features are fused using Concatenation method in level 2 and these multi-level fused vector data is set as an input for neural network and results are plotted. Neural network model concerning input neuron $1: 2,1: 3$, and 1:4 ratio hidden neurons models are implemented. Extending the experimentation neural network model with multiple hidden layers of 756 hidden neurons in the first layer and 256 hidden neurons in the second layer is implemented and results are plotted.

\section{RESULTS}

The results are based on Level 1 feature fusion: The experiment is conducted on unimodal biometrics. Original database and upsampled database are fused using the concatenation technique, and the concatenated feature vector is set as an input for the neural network. The classification accuracy is given in Table 2. The results are based on level 2 feature fusion: signature and fingerprint feature vector obtained from level 1 SUM, MAX, MIN, PRODUCT, and concatenation technique fusion is fused in level 2 using concatenation technique. Feature vector obtained after both level 1 and level 2 fusions is set as an input for neural network and results are plotted. The classification accuracy of the neural network model is given in Table 3. 
Table 2. Unimodal system: single level feature fusion

\begin{tabular}{lcc}
\multicolumn{1}{c}{ Biometric traits } & Fusion technique & $\begin{array}{l}\text { Neural Network Model } \\
\text { Classification accuracy }\end{array}$ \\
\hline $\begin{array}{l}\text { MCYT Standard Signature data sample } \\
\text { and 1:2 ratio up sampled data. }\end{array}$ & Concatenation & $85 \%$ \\
$\begin{array}{l}\text { SDUMLA Standard fingerprint data } \\
\text { sample and 1:2 ratio up sampled data }\end{array}$ & Concatenation & $60 \%$ \\
\hline
\end{tabular}

Table 3. Multimodal System: multi-level feature fusion

\begin{tabular}{llc}
\hline \multicolumn{1}{c}{ Biometric traits } & \multicolumn{1}{c}{ Multi-Level Fusion technique } & \multicolumn{1}{c}{$\begin{array}{c}\text { Neural Network Model } \\
\text { Classification accuracy }\end{array}$} \\
\hline & Level 1: Concatenation & $91.66 \%$ \\
& Level 2: Concatenation & Level 1: SUM \\
MCYT Standard Signature data sample and & Level 2: Concatenation & $92.5 \%$ \\
1:2 ratio up sampled data. & Level 1: MAX & $91.66 \%$ \\
SDUMLA Standard fingerprint data sample & Level 2: Concatenation & \\
and 1:2 ratio up sampled data & Level 1: MIN & $92.0 \%$ \\
Level1: Unimodal biometrics & Level 2: Concatenation & \\
Level2: Multimodal Biometrics & Level 1: Product & $93.33 \%$ \\
& Level 2: Concatenation & \\
\hline
\end{tabular}

Figure 5 shows the classification accuracy of each network model with different fusion techniques used in both level 1 and level 2 fusions. Figure 5 shows the plot for data available in Table 3, the x-axis shows fusion techniques at both level 1 and level 2 respectively and, the y-axis shows classification accuracy. Results based on varying hidden neurons: From Table 3 it has been observed that the level 1 product method and level 2 concatenation methods showed good results, that results are obtained by developing four neural network models by varying hidden neurons. The results of all four models are given in Table 4.

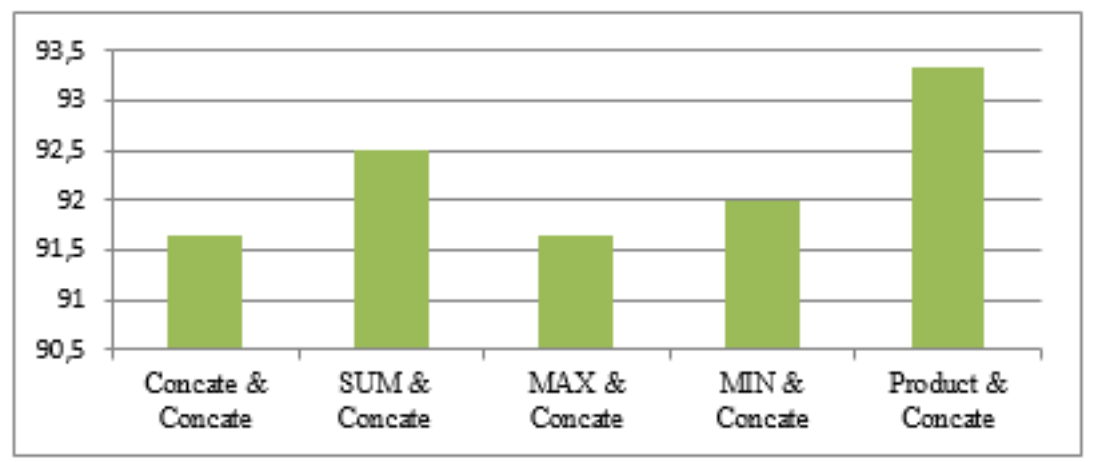

Figure 5. Multi-level feature fusion techniques vs neural network model classification accuracy

Table 4. Multimodal System: Multi-level feature fusion for different hidden neuron

\begin{tabular}{llc}
\hline \multicolumn{1}{c}{ Biometric traits } & Hidden Neurons & $\begin{array}{c}\text { Neural Network Model } \\
\text { Classification accuracy }\end{array}$ \\
\hline $\begin{array}{l}\text { MCYT Standard Signature data sample and } \\
\text { 1:2 ratio up sampled data. }\end{array}$ & 252 Neurons & $92.00 \%$ \\
$\begin{array}{l}\text { SDUMLA Standard fingerprint data sample } \\
\text { and 1:2 ratio up sampled data }\end{array}$ & 504 Neurons & $92.50 \%$ \\
$\begin{array}{l}\text { Level1: Unimodal biometrics (Product) } \\
\text { Level2: Multimodal Biometrics } \\
\text { (Concatenation) }\end{array}$ & 756 Neurons & $93.33 \%$ \\
\hline
\end{tabular}

Figure 6 shows the classification accuracy of the network model by varying hidden neurons used in the hidden layer's $\mathrm{x}$-axis shows various neurons used in the hidden layers, the $\mathrm{y}$-axis shows classification 
accuracy. Results based on varying hidden layers: From Table 4 it has been observed 756 hidden neurons showed good results, the same neural network model is extended by adding multiple hidden layers. The results of the single hidden layer with 756 hidden neurons and multiple hidden layers with 756 hidden neurons are given in Table 5. In the literature survey many works show more than $95 \%$ classification accuracy, but for very limited data sets. In the proposed work $93.33 \%$ accuracy was achieved for larger data sets. From the literature it has been observed that, no other work is proposed with the fusion of fingerprint and signature biometric traits at multi-level with deeplearning model for classification. When compared with unimodal fringerprint, in proposed model recognition rate is increased by $8.33 \%$ and for signature samples proposed model recognition rate is increased by $33.33 \%$.

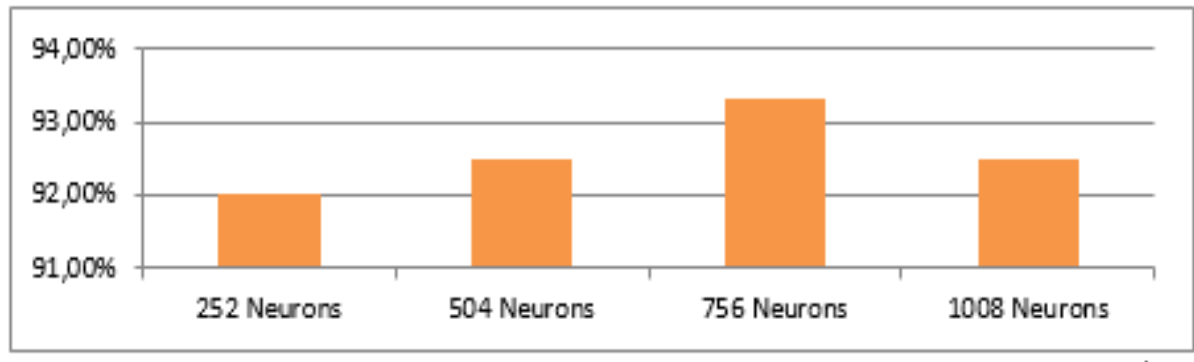

Figure 6. Hidden neurons vs neural network model classification accuracy

Table 5. Multimodal System: Multi-level feature fusion for different hidden layers

\begin{tabular}{lll}
\hline \multicolumn{1}{c}{ Biometric traits } & Hidden Layer & $\begin{array}{l}\text { Neural Network Model } \\
\text { Classification accuracy }\end{array}$ \\
\hline $\begin{array}{l}\text { MCYT Standard Signature data sample and } \\
\text { 1:2 ratio up sampled data. }\end{array}$ & $\begin{array}{l}756 \text { Neurons } \\
\text { (single layer) }\end{array}$ & $93.33 \%$ \\
$\begin{array}{l}\text { SDUMLA Standard fingerprint data sample } \\
\text { and 1:2 ratio up sampled data }\end{array}$ & $\begin{array}{l}756 \text { Neurons } \\
\text { (first layer) } \\
\text { Level1: Unimodal biometrics (concatenation) }\end{array}$ & $\begin{array}{l}\text { 252 Neurons } \\
\text { (second layer) }\end{array}$ \\
\hline
\end{tabular}

\section{CONCLUSION}

In this paper, several experiments are carried out by varying hidden neurons and hidden layers of the neural network model. By examining the entire neural network model best models are selected and results are explored. In the proposed method multilevel feature fusion for an upsampled database is implemented by using a deep neural network model. The main idea of this work is to obtain better classification accuracy in multimodal signature and fingerprint biometric. However, there will be no fixed method to design deep learning networks like how to choose hidden neurons, hidden layers, activation function, etc. From the experiment, it has been observed that the proposed model increases the classification accuracy of multimodal biometrics over unimodal biometrics recognition for larger data sets. In Multilevel feature fusion combination of concatenation and product operations showed a better result of $93.33 \%$ of classification accuracy when experimented on a single layer with 756 hidden neurons.

\section{REFERENCES}

[1] M. Oloyede, G. P. Hancke, "Unimodal and multimodal biometric sensing systems: A Review," IEEE Access 2016, vol. 4, pp. 7532-7555, Sep.2016, doi: 10.1109/ACCESS.2016.2614720.

[2] Shaheed, et al., "A systematic review of finger vein recognition techniques." Informatics, vol.9, pp. 213, Aug. 2018, doi: 10.3390/info9090213.

[3] Pooja BS and Arjun BC, "Face recognition using artificial neural networks (ANN)" National Conference on Recent Advances in Information \& Communication Engineering, Feb. 2016, doi: 10.1109/SPIN48934.2020.9071378.

[4] Chandan Rani and Arjun BC “An efficient signature based biometric system using BPNN," International Journal of Innovative Research and Advanced Studies (IJIRAS), vol. 3, no. 6, May 2016.

[5] Ding, et al., "Robust face recognition via multimodal deep face representation." IEEE Trans. Multimed, vol.17, pp. 2049-2058, 2015.

[6] Alaa S. Al-Waisy, R. Qahwaji, S. S. Ipson, S. Al-Fahdawi, "A multi-biometric iris recognition system based on a deep learning approach,” Pattern Anal. Appl. vol. 21, pp. 783-802, Oct. 2018. 
[7] W. Liu, L. Sun, L. Zhang, L. Zhang, "Finger vein recognition based on deep learning," Proceedings of the 2017 12th IEEE Conference on Industrial Electronics and Applications, Siem Reap, Cambodia, pp. 205-210, Jun. 2017.

[8] K. Soumia, B. Mohammed, H. Aymen, K. Ibrahim, "Biometric authentication using curvelet transform," Indonesian Journal of Electrical Engineering and Computer Science (IJEECS), vol. 20, no. 3, pp. 1332-1341, Dec. 2020, doi: 10.11591/ijeecs.v20i3.

[9] Haider, et al., "A Survey of Biometric Recognition Using Deep Learning” EAI Endorsed Transactions on Energy Web Online First published, Oct. 2020.

[10] Tiong, et al., "Multimodal face biometrics by using convolutional neural network," Journal of Korea Multimedia Society, vol. 20, no. 2, pp. 170-8, 2017, doi: 10.9717/kmms.2017.20.2.170.

[11] Jiajia Geng, Jiajia Geng, Yiu-ming Cheung, "Audio-visual speaker recognition via multi-modal correlated neural network," 2016 IEEE/WIC/ACM International Conference onWeb Intelligence Workshops (WIW), Oct. 2016, doi: 10.1109/WIW.2016.043.

[12] Navdeep, et al., "A novel multi-model biometricfusion approach using palm-print \& face biometric," International Journal of Latest Trends in Engineering andTechnology, vol. 8, no.3, pp. 240-247, 2017, doi: 10.1109/ARTCom.2009.224.

[13] Priya et al., "Multimodal biometric authentication using back propagation artificial neural network." International Journal of Simulation: Systems, Scienceand Technology, vol. 19, no. 6, pp.1-8, 2018.

[14] P. Silva, E. J.da Silva Luz,L. A. Zanlorensi, D. Menotti," Multimodal feature level fusion based on particleswarm optimization with deep transfer learning,” 2018 IEEECongress on Evolutionary Computation (CEC), Jul. 2018.

[15] Sukhdev Singh and Chander Kant, "FKP and iris based multimodal biometric system using PCA with NFNN," International Conference on Sustainable Computing in Science, Technology and Management (SUSCOM), Feb. 2019.

[16] El Mehdi Cherratv, Rachid Alaoui, Hassane Bouzahir, "Score fusion offinger vein and face for human recognition based onconvolutional neural network model”. International Journal of Computing, vol. 19, no. 1, pp. 11-19, Mar. 2020.

[17] P. Kumari, K. R. Seeja, "Periocular biometrics for non-ideal images: with off-the-shelf Deep CNN \& Transfer Learningapproach”. Procedia Computer Science, vol. 167, pp. 344-352, 2020.

[18] Arun A. Ross, K. Nandakumar, A, K, Jain, "Handbook of Multibiometrics," Springer International Edition, 2016.

[19] Yilong, et al., "A multimodal biometric database". The 6th Chinese Conference on Biometric Recognition (CCBR 2011), LNCS 7098, 2011, pp. 260-268, doi: 10.1007/s10044-009-0151-4.

[20] J. Ortega-Garcia, "MCYT base line corpus: a bimodal biometric database" IEE proc.-Vis image signal process, vol 150, no. 6, Dec. 2103, doi: 10.1049/ip-vis:20031078.

[21] Arjun B C, H N. Prakash, "Feature level fusion of seven neighbor bilinear interpolation data sets of finger vein and iris for multimodal biometric recognition," International Journal of Advanced Trends in Computer Science and Engineering, vol. 9, no.2, 2020, doi: 10.30534/ijatcse/2020/95922020.

[22] El mehdi Cherrat, Rachid Alaoui, Hassane Bouzahir, "A multimodal biometric identification system based on cascade advanced of fingerprint, fingervein and face images," Indonesian Journal of Electrical Engineering and Computer Science, vol. 18, no, 1, pp. 1562-1570 Apr. 2020, doi: 10.11591/ijeecs.v18.i1.

[23] Arjun B C, H N Prakash, "Multimodal biometric recognition: fusion of modified adaptive bilinear interpolation data samples of face and signature using local binary pattern features" International Journal of Engineering and Advanced Technology (IJEAT), Vol. 9, no. 3, pp. 2249-8958, Feb. 2020, doi: 10.35940/ijeat.C6117.029320.

[24] Jason Brownlee, "Deep learning with python develop deep learning models on theano and tensorflow," Machine Learning Mastery, 2016.

[25] A. Ross, Govindarajan, "Feature Level fusion using hand and face biometrics," Proceedings of SPIE Conference on Biometric Technology for Human Identification I, vol. 5779, pp. 196-204, 2005, doi: 10.1117/12.606093.

[26] Ross A, "Fusion, Feature-Level," Encyclopedia of Biometrics. Springer, Boston, 2009.

[27] N. Dalal, B. Triggs, "Histograms of oriented gradients for human detection," IEEE Computer Society Conference on Computer Vision and Pattern Recognition, vol. 1, pp. 886-893, Jun. 2015, doi: 10.1109/CVPR.2005.177.

\section{BIOGRAPHIES OF AUTHORS}

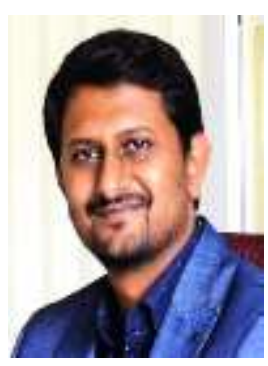

Arjun B C received a degree in Bachelor of Computer Science and Engineering from Visvesvaraya Technological University, Belgaum, Karnataka, India, and M. Tech in Industrial Automation and Robotics from Visvesvaraya Technological University Belgaum, Karnataka. India. Currently, he is pursuing a Ph.D. in Computer Science and Engineering at Visvesvaraya Technological University, Belgaum Karnataka, India. He is currently working as an Assistant Professor in the Department of Information Science \& Engineering at Rajeev Institute of Technology, Hassan Karnataka, and India. He has 2 years of industry experience and 10 years of teaching experience. His research interest includes Image processing, Pattern recognition, Artificial Intelligence, Artificial Neural Network, Deep Learning, and Machine Learning. He has published papers in conferences and International Journals. 
Dr. H N Prakash received a degree in Bachelor of Electronics and Communications and Engineering from Mysore University, Karnataka, India, and $M$. Tech in Electronics Instrumentation from National Institute of Technology Warangal, Telangana, India, and Ph.D in Computer Science from Mysore University, Karnataka, India. He is currently working as a Professor and Head in the Department of Computer Science \& Engineering at Rajeev Institute of Technology, Hassan, Karnataka, India. He has 30 years of teaching experience. His research interest includes image processing, pattern recognition, Artificial Intelligence, Artificial Neural Network and Machine Learning. He has published many papers in National and International conferences and Journals. He has published IEEE transactions in Pattern Recognition and Machine Intelligence. 